\title{
A Flux-based Inverse Integral Formulation for Steel Shell Magnetization Identification
}

\author{
G. Chavin-Collin ${ }^{\mathrm{a}, \mathrm{b}}$, B. Bannwarth ${ }^{\mathrm{a}}$, D. Cavallera ${ }^{\mathrm{b}}$, O. Chadebec $^{\mathrm{a}, *}, \mathrm{~N}$. \\ Galopin $^{\mathrm{a}}$, G. Meunier ${ }^{\mathrm{a}}$, O. Pinaud ${ }^{\mathrm{a}}$, L-L. Rouve ${ }^{\mathrm{a}}$ \\ ${ }^{a}$ Univ. Grenoble Alpes, CNRS, Grenoble-INP, G2Elab, F-38000 Grenoble, France \\ ${ }^{b}$ Naval Group, F-75732 Paris, France
}

\begin{abstract}
A new algorithm for the identification of the permanent magnetization of ferromagnetic sheets is proposed. From magnetic measurements close to the ferromagnetic material, the magnetization distribution is reconstructed. The forward problem involves an integral formulation based on the interpolation of the flux density in the sheet, leading to a linear matrix system linking the magnetization to the measurements. The inverse problem is solved with a balanced singular values decomposition in order to stabilize the solution. The effectiveness of the method is demonstrated using both a numerical test case and an experimental validation.
\end{abstract}

Keywords: Magnetization identification, magnetostatic inverse problem, volume integral method, thin magnetic shell

\section{Introduction}

Defense ships and submarines are made of steels which is a ferromagnetic material. Steel acquires a magnetization in the combined presence of the earth's magnetic field and the water pressure (magneto-elasticity effect). The steel 5 magnetization generates a magnetic signature around the ship, that may lead to its destruction by magnetic mines or to its localization by aircrafts embedding

\footnotetext{
* Corresponding author

Email address: olivier.chadebec@g2elab.grenoble-inp.fr (O. Chadebec)
} 
high accuracy magnetic sensors. To control the magnetic risk, it is essential to accurately identify the ship magnetization, and then to compensate for the associated signature by adjusting currents flowing in degaussing coils installed in the ship. This paper proposes a new identification algorithm to enable a ship to self-determine its own magnetization during navigation and thus achieve a closed loop degaussing system.

A magnetic sheet, which represents the ship hull, has two kinds of magnetization. The induced one is the reversible magnetic reaction of the material to an inductor field (i.e. the earth's magnetic field). Induced magnetization is deterministic: it is easily computed as the magnetic constitutive law of the material and the ship geometry are generally well known. The case of the permanent magnetization is more problematic because it depends on the magnetic history of the sheet, which is impossible to know and model. As we have no idea of this history, the permanent magnetization state determination requires the use of external magnetic measurements made on magnetic sensors, placed close to the hull on-board the ship as well as the solving of an inverse problem.

Many works dealing with magnetization identification can be found in the literature aiming to reconstruct magnetization of magnetic rollers [1], electrical machine magnets [2] and also navy ship hulls [3, 4, 5, 6]. In most of these works, the forward problem is formulated thanks to an integral relation linking the magnetization to the magnetic field produced in air on an external sensor. The magnetic region is meshed in finite elements. A set of shape functions is chosen on this mesh to approximate the unknown magnetization distribution. in the air on one sensor to the magnetization degrees of freedom. By setting several magnetic sensors around the magnetic region, a linear matrix system is obtained which form the inverse problem. The inverse problem being ill-posed, some regularization techniques or additional information have to be used in order to ensure the uniqueness and the stability of the solution.

In order to interpolate the magnetization, 0-order vector shape functions can be used like in the well-known Magnetic Moments Method (MMM). With 
such an interpolation, magnetization of volume regions has been reconstructed for 2D [1] or 3D [2] problems. For ferromagnetic sheet, a similar approach has been extended to the identification of the tangential components of the magnetization [4. An alternative can be the use of the scalar magnetic potential as the unknown quantity on the surface region [5].

All these formulations of the inverse problem are obviously ill-posed. The Tikhonov's regularization is often used in order to stabilize the solution but 45 the choice of the weighting parameter is always a difficulty [1, 3]. To avoid the problem of choosing a regularization parameter, it has been proposed to reduce the solution searching space by introducing the constitutive magnetic law of the material in the inverse formulation and by using a singular value decomposition (SVD) in order to solve the linear matrix system [4. This approach has shown 50 a very good efficiency with only a limited number of sensors but located not too close from the hull. This limitation is an important drawback because for a closed-loop degaussing system, on-board sensors are mainly placed very close to the hull. This poor efficiency for close measurements can be associated to the lack of accuracy of MMM which is based on 0-order shape functions for the magnetization and leads to artificial numerical singularity of the field in the vicinity of the sources. Another way to solve the inverse problem is to formulate it as a non-linear constrained optimization problem. The use of the adjoint variable method enables the determination of equivalent charges or dipoles located on the mesh of the hull and then to get a model of the permanent magnetization [6, 7]. These methods have been validated for sensors far from the shell but nothing has been demonstrated with close measurements. Moreover, a regularization process is still needed.

This paper proposes a new inversion method based on a forward integral formulation recently proposed in (Ref. [8]). It has been demonstrated that this integral formulation is very accurate even if the field has to be computed really close to the sheet because the magnetization is considered as linear on surfaces element. This point is a great advantage compared to our former forward model used in (Ref. 4]) where the magnetization is uniform in each element, generating 
important numerical inaccuracies close to the shell and constraining the sensor

mapping. It will be shown in the following that the new inversion process leads to accurate results with only a few sensors located close to the magnetized structure and without the choice of any empirical regularization parameter. Once the new method efficiency demonstrated with a numerical example, experimental measurements made close to a physical mock-up (hollow ferromagnetic cylinder) will validate the inversion process and show how the approach can be applied to the evaluation of magnetic anomaly created by navy ships or submarines in order to reduce the risk of detection or de-struction by magnetic mines.

This paper is structured as follows: the second part is dedicated to the forward modelling. The third part will introduce the resolution of the inverse so problem. The fourth part will present both numerical and experimental results. The paper will end with conclusions.

\section{Forward Modelling}

The integral forward formulation of the magnetostatic problem is detailed in (Ref. [8]) but the most points are reminded in this section. Let us consider a magnetic problem composed of a nonconductive ferromagnetic region $\Omega$ with a reluctivity $\nu$ and placed in a static low-level inductor magnetic field $\mathbf{H}_{0}$. The level of the inductor field being low, it is classical to assume that the magnetic material is linear. Let us assume that the magnetic material has a permanent magnetic state, which can be represented by a coercive field $\mathbf{H}_{c}$. The constitutive equation linking the total field $\mathbf{H}$ to the induction $\mathbf{B}$ is:

$$
\mathbf{H}=\nu \mathbf{B}-\mathbf{H}_{c} .
$$

In the whole domain, the total field can be written as the sum of $\mathbf{H}_{0}$ and the reduced field $\mathbf{H}_{\text {red }}$ due to the magnetization $\mathbf{M}$, which is the total magnetization. The reduced field $\mathbf{H}_{\text {red }}$ derives from a scalar reduced potential $\phi_{\text {red }}$.

$$
\mathbf{H}=\mathbf{H}_{0}+\mathbf{H}_{\text {red }}=\mathbf{H}_{0}-\nabla \phi_{\text {red }} .
$$


Applying the Helmholtz decomposition, an integral expression for the scalar potential $\phi_{\text {red }}$ is obtained:

$$
\phi_{\text {red }}=-\frac{1}{4 \pi} \int_{\Omega} \mathbf{M} \cdot \nabla G d \Omega
$$

where $G$ is the standard 3D Green's function (i.e. the inverse of the distance between the point where the scalar potential is expressed and the integration point) and $\mathbf{M}$ is the magnetization of the material such as $\mathbf{M}=\left(\nu_{0}-\nu\right) \mathbf{B}+\mathbf{H}_{c}$. Equations (1) and (2) combined with the integral expression of $\phi_{\text {red }}$ (3) lead to the magnetostatic integral equation with the induction $\mathbf{B}$ as state variable 9 :

$$
\nu \mathbf{B}-\frac{1}{4 \pi} \int_{\Omega}\left(\left(\nu_{0}-\nu\right) \mathbf{B}+\mathbf{H}_{c}\right) \cdot \nabla G d \Omega=\mathbf{H}_{0}+\mathbf{H}_{c} .
$$

In the case of a magnetic sheet, the approximation of thin element simplifies a volume problem into a surface one by considering that the magnetic induction in the active region is uniform across the thickness and is tangential to the sheet. In (Ref. [8]), it has been shown that it is appropriate to interpolate B with Whitney 2-form shape functions (also known as face shape functions) and to express it with a linear combination of magnetic flux $\Phi_{B}$ flowing in the sheet through the equivalent faces of the meshed surface (i.e. the edges of the equivalent surface elements). Equation (4) needs to be completed in order to enforce the free-divergence of the flux. A solution is to express (4) in the basis of independent flux loops $\boldsymbol{\Phi}_{B I}$. Let us note $\mathbf{P}$ the incidence matrix linking the flux flowing in each face of the mesh to the independent ones. Applying a Galerkin's projection method to (4), a linear system of equations whose unknowns are independent fluxes is achieved.

$$
\mathbf{P}\left[\begin{array}{cc}
\mathbf{R} & \mathbf{0} \\
\mathbf{0} & \mathbf{L}
\end{array}\right] \mathbf{P}^{t} \boldsymbol{\Phi}_{B I}=\mathbf{P}\left(\begin{array}{l}
\mathbf{U} \\
\mathbf{0}
\end{array}\right),
$$

where $\mathbf{R} \in \mathbb{R}^{n_{f} \times n_{f}}, \mathbf{U} \in \mathbb{R}^{n_{f} \times 1}, \mathbf{L} \in \mathbb{R}^{n_{e} \times n_{e}}, \mathbf{P} \in \mathbb{R}^{\left(n_{f}+n_{e}\right) \times n_{B I}}, \mathbf{\Phi}_{B I} \in$ $\mathbb{R}^{n_{B I} \times 1}, n_{f}$ is the number of facet element (i.e. edge of the surface mesh elements), $n_{e}$ is the number of surface elements and $n_{B I}$ is the number of independent fluxes. Expressions of the matrix terms are:

$$
R_{i j}=\frac{1}{e} \int_{S} \nu \mathbf{w}_{i} \cdot \mathbf{w}_{j} d S
$$




$$
\begin{gathered}
L_{i j}=\frac{1}{4 \pi} \frac{\nu_{0}-\nu}{S_{e i} S_{e j}} \int_{S_{e i}} \int_{S_{e j}} G d S_{e i} d S_{e j}, \\
U_{i}=\int_{S} \mathbf{w}_{i} \cdot\left(\mathbf{H}_{0}+\mathbf{H}_{c}\right) d S \\
+\frac{1}{4 \pi} \frac{1}{S_{e i}} \sum_{j} \int_{S_{e i}} \int_{S_{e j}} \Delta H_{c n} G d S_{e i} d S_{e j},
\end{gathered}
$$

where $S$ is the averaged geometric surface representing the $\omega$ volume region, $e$ is the thickness of the sheet, $S_{e i}$ is the surface of the element $i, \nu_{j}$ is the reluctivity

85 $\Delta H_{c n}$ is the jump of the normal component of the coercive field between two adjacent elements. It can be noted that the last double integral term in (8) can be neglected considering that the cross-section of the shell is much smaller than the other dimensions. Thus, the matrix system (5) can be rewritten in a more compact expression:

$$
\mathbf{M} \boldsymbol{\Phi}_{B I}=\mathbf{S}_{\mathbf{H}_{0}}+\mathbf{S}_{\mathbf{H}_{c}},
$$

where $\mathbf{M} \in \mathbb{R}^{n_{B I} \times n_{B I}}, \mathbf{S}_{\mathbf{H}_{0}} \in \mathbb{R}^{n_{B I} \times 1}$ and $\mathbf{S}_{\mathbf{H}_{c}} \in \mathbb{R}^{n_{B I} \times 1}$.

Let us remind that in the forward problem (9), both coercive and inductor fields are known as well as the reversible reluctivity of the material. However, in the context of an inverse problem, the point of view is different. The inductor field as well as the reversible permeability are known but the magnetic state in a null inductor field is unknown. In other words, $\mathbf{H}_{c}$ has to be determined for each element of the mesh and considered as an unknown of the problem. This is why external magnetic measurements have to be added in order to provide additional information to the system.

\section{Inverse Problem Formulation}

An equation linking the flux distribution in the shell to the induction measured on external magnetic sensors has to be added. The magnetic field in air 
is 8 :

$$
\mathbf{B}_{\text {sensor }}=\nabla \int_{S}\left(1-\frac{\nu}{\nu_{0}}\right) \nabla_{S} \cdot \mathbf{B} G d S+\mathbf{B}_{0},
$$

where $\mathbf{B}_{\text {sensor }}$ is the induction measured on a magnetic sensor located in the be taken into account.

Let us remember that $\mathbf{H}_{c}$ is an unknown of the problem. To determine it numerically, an interpolation function space has to be selected. A good choice can be also the face shape functions. Thus, the last term in $(9)$ can be replaced 125 by the product of a matrix $\mathbf{N}$ and the unknowns $\boldsymbol{\Phi}_{H_{c}}$ associated to $\mathbf{H}_{c}$ explicit, leading to a new matrix system (12) which replaces $(9)$.

$$
\mathbf{M} \Phi_{B I}-\mathbf{N} \Phi_{H_{c}}=\mathbf{S}_{H_{0}},
$$


where $\mathbf{N} \in \mathbb{R}^{n_{B I} \times n_{f}}$.

By combining (11) and (12) and after some algebra, the final matrix system with $\boldsymbol{\Phi}_{H_{c}}$ as unknown is obtained:

$$
\mathbf{A} \mathbf{M}^{-1} \mathbf{N} \Phi_{H_{c}}=\left(\mathbf{B}_{m e s}-\mathbf{A} \mathbf{M}^{-1} \mathbf{S}_{H_{0}}\right)
$$

$$
\mathbf{Z} \Phi_{H_{c}}=\mathbf{T}
$$

System (14) is a more compact writing of 130 . It must be pointed out that (14) includes both the information from the magnetic measurement and the magnetic behavior of the material. The solution of (14) is non-unique, the system being underdetermined since the number of equations is far smaller than the number of unknowns, due to a limited experimental set of measurement values. The singular value decomposition makes it possible to compute the Moore-Penrose pseudo-inverse $\mathbf{Z}^{+}$of $\mathbf{Z}$ [10]:

$$
\mathbf{\Phi}_{H_{c}}=\mathbf{Z}^{+} \mathbf{T} .
$$

The condition number of the $\mathbf{Z}$ matrix is the ratio between the highest and the lowest singular values. The higher is the ratio the worst is the conditioning. Before solving (14), a "row and column" balancing [11] is applied to equilibrate the influence of each magnetization sources on the induction measurements. Mathematically, it consists in dividing each element of the columns by the norm of the concerned column, the same operation being also proceeded for the lines. Several iterations of this process make it possible to improve the condition number of the matrix $\mathbf{Z}$. This process can be seen as a preconditioning technique. If this preconditioning technique is not enough efficient, the principle of the spectrum truncation can be used [10]: it consists in removing the smallest singular values in order to limit the influence of the numerical noise. 


\section{Validations and Results}

\subsection{Numerical Validation}

ferromagnetic plate is considered and a permanent magnetization is applied as follows. Firstly, a material with an anhysteretic reluctivity $\nu_{a}$ and a null coercive field is placed in a homogenous inductor field $\mathbf{H}_{0_{1}}=(1,1,0)(\mathrm{A} / \mathrm{m})$. Its magnetic state is computed by solving $(9)$, leading to $(\mathbf{H}, \mathbf{B})$. Then, for each in order to compute the $\mathbf{H}_{c}$ field for each element. In a second step, $(9)$ is solved again with a new inductor field $\mathbf{H}_{0_{2}}=(1,0,0)(A / m)$ and the previously computed $\mathbf{H}_{c}$ field in order to get a complex magnetic state mixing permanent and reversible magnetizations. The numerical measurements are generated with

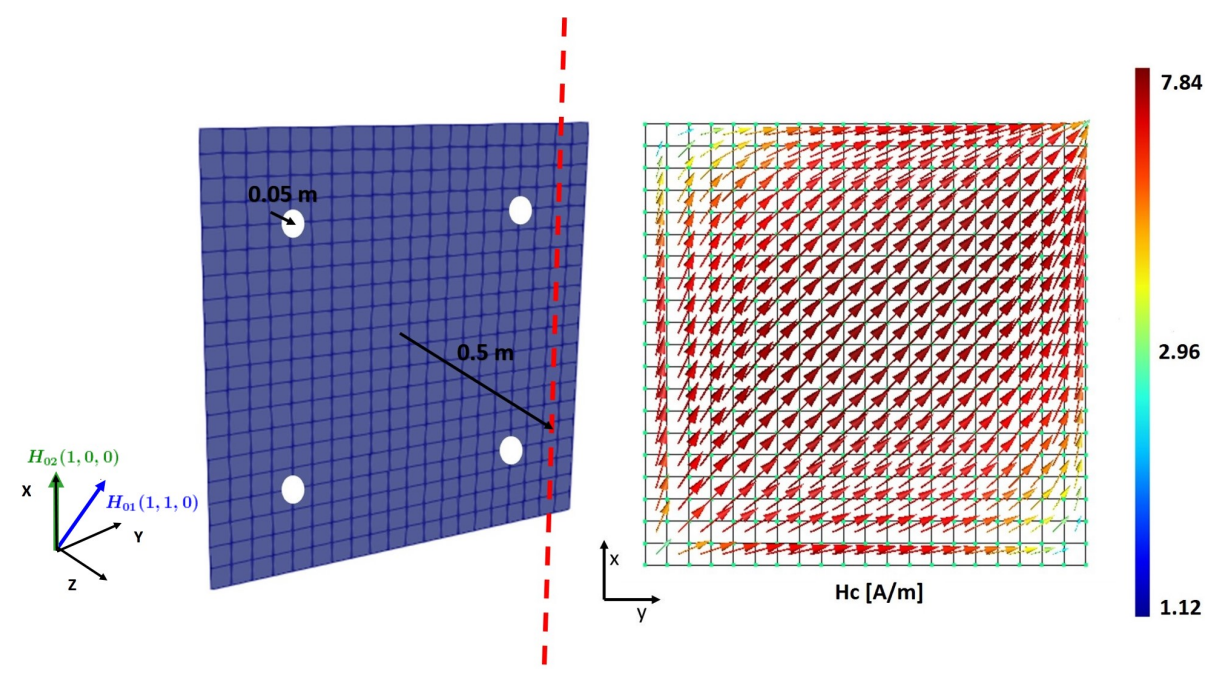

Figure 1: Test case: Iron sheet mesh and inversion test configuration (dimensions: $1 \mathrm{~m} \times 1 \mathrm{~m}$, thickness: $2 \mathrm{~mm}$, number of elements: 440, number of unknowns: 760,4 tri-axis sensors represented by white dots located at $5 \mathrm{~cm}$ from the sheet). The extrapolated field is computed on the red dashed line. Left: mesh and sensors location. Right: Non trivial $\mathbf{H}_{c}$ distribution $(A / m)$ to reconstruct.

The purpose of the test is to solve the inverse problem from the numerical 
measurements and to identify the magnetic state of the sheet. The problem is strongly underdetermined because only 12 equations $(4 \times 3$ components for the measured induction) should enable the determination of 760 unknowns. Firstly, the system (15) is solved with a simple balanced SVD without spectrum truncation. Figure 2 shows the result of the identified repartition of $\mathbf{H}_{c}$.

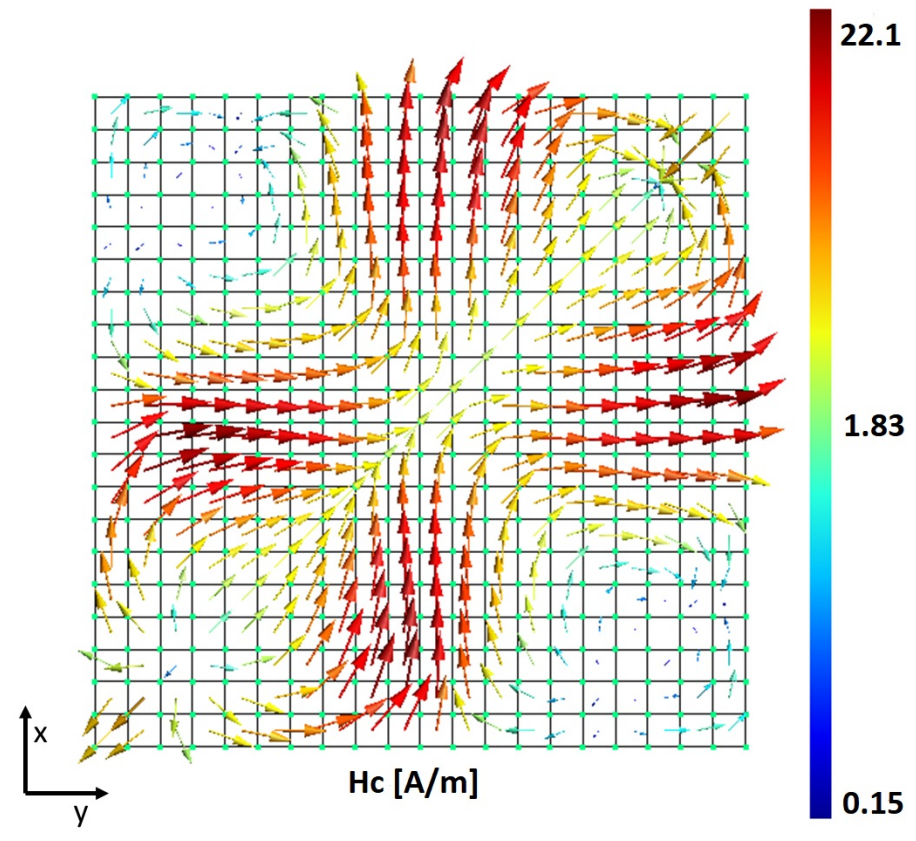

Figure 2: Irregular identified $\mathbf{H}_{c}$ distribution obtained by solving 14 with a balanced SVD. The solution has major spatial irregularities that are clearly unrealistic.

The result obtained is very unsatisfying. In fact, the $\mathbf{H}_{c}$ distribution identified is highly irregular and differs strongly from the expected distribution represented in Fig. 1. The problem being underdetermined, the solution is naturally non-unique and (15) leads to a flux distribution that fits magnetic measurements but without physical sense. Classically, a solution would be to use a Tikhonov's regularization but an alternative approach is proposed here.

Mathematically, the SVD returns the solution with the minimal norm. So, 
the previous solution is the minimal $\mathbf{H}_{c}$ fluxes flowing through the shell which fit the measurements. This minimal flux solution does not make sense physically. A better option would be to opt for a solution that ensures the flux regularity. At this stage, it is therefore necessary to change the unknowns of the problem. Let us consider the surface divergence of the coercive field on the shell such as:

$$
\nabla_{S} \cdot \mathbf{H}_{c}=Q_{H_{c}} .
$$

Note that the divergence can be computed naturally because it is associated The results obtained are presented in Fig. 4. The target field (solid line, no marker) is computed with the real initial magnetization and the predicted field (dashed line, diamond markers) is computed with the identified magnetization. 
Both curves are very close to each other attesting that the magnetization has been correctly predicted. The error is defined as the norm of the difference between the real value and the extrapolated value divided by the max of the real value for each component. The mean error is around $1 \%$ for all induction components (see Tab. 11. Let us notice that the proposed test case is a quite difficult one because the geometry presents high peak effects at the four corners of the plate. However, the algorithm tested with only 4 tri-axis sensors (i.e. 12 measurement equations compared to the 760 unknowns) remains very good in terms of performance.

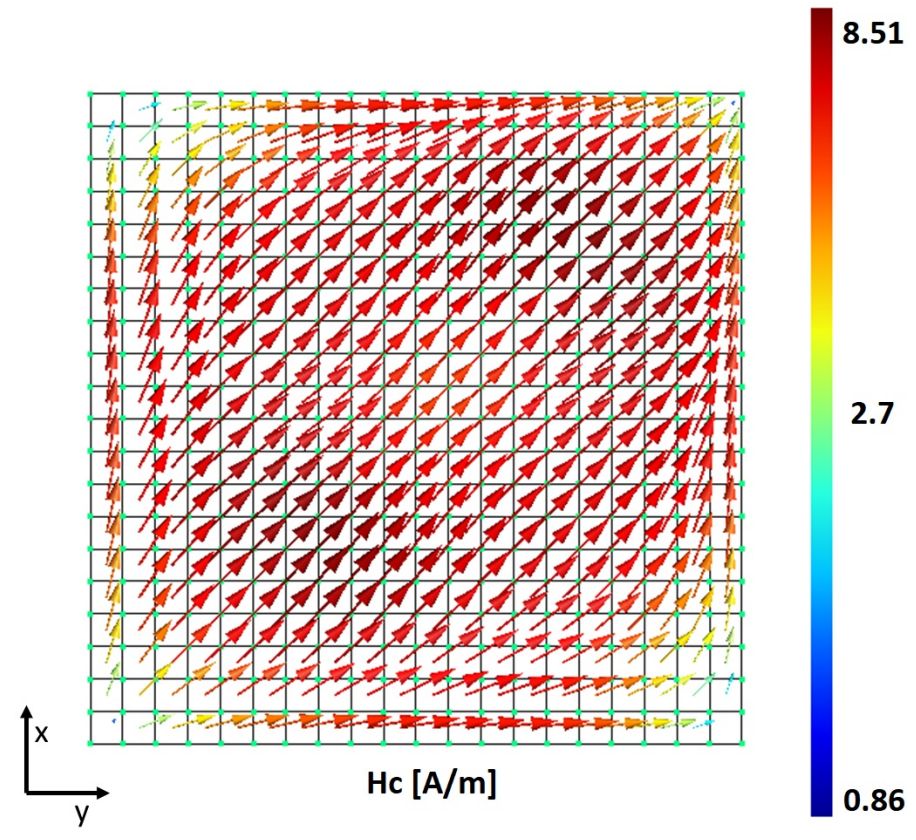

Figure 3: Smooth and acceptable identified $\mathbf{H}_{c}$ using fictive charge inversion based on the solution of 17 . 
Table 1: Computed errors of the reconstructed induction.

\begin{tabular}{cccc}
\hline & $X$ component & $Y$ component & $Z$ component \\
\hline max error (\%) & 2.25 & 2.26 & 1.65 \\
mean error (\%) & 0.42 & 1.02 & 0.45 \\
\hline
\end{tabular}

\subsection{Experimental Validations}

\subsubsection{Description of the measurement set-up}

An experimental set-up has been developed to validate the identification algorithm. A hollow ferromagnetic cylinder $(0.5 \mathrm{~m}$ long, $59 \mathrm{~mm}$ of external radius, $2 \mathrm{~mm}$ of thickness) filled with hydraulic oil, is subjected to an internal pressure up to $10 M P a$, driven by an external pump. The cylinder is wound to allow its demagnetization (Fig. 5). Fourteen Fluxgate biaxial magnetic sensors are arranged $1 \mathrm{~cm}$ far from the steel on 2 lines. This set of sensors consists of 7 vertical cores, 7 transverse cores and 14 longitudinal cores (Fig. 6). The prototype is placed in a large magnetic field simulator that has been designed to create any field from zero up to $70 \mu T$ in various directions thanks to a triaxial set of coils, driven by current generators. The cylinder is mounted on a trolley driven over a pair of rails by an electrical motor along the longitudinal direction. It allows magnetic signature measurements on a $[-1.7 \mathrm{~m}, 1.7 \mathrm{~m}]$ line on two fixed magnetic sensors (Fig. 7): sensor $S_{1}$ is located at $10 \mathrm{~cm}$ below the axis of the cylinder ("near field" extrapolation), sensor $S_{2}$ is placed further, at $1 m$ ("far field" extrapolation).

This set-up has been developed in order to study magneto-elastic effects i.e. the variation of the permanent magnetization versus the combined effect of a low magnetic inductor field and a high-pressure acting on the steel [12]. Consequently, the conferred permanent state is relevant to illustrate the phenomenon undergone by submarine hulls. 


\subsubsection{Permanent longitudinal magnetization}

The cylinder is initially demagnetized. Then an axial uniform magnetic induction of $40 \mu T$ is applied with the field simulator and the mock-up is subjected to an internal pressure of $10 \mathrm{MPa}$. Finally, the pressure is decreased to zero. efficiency of the proposed method.

\subsubsection{Composed magnetization identification}

In a second experimentation, a more complex magnetic state identification is proposed. The cylinder is initially demagnetized. Then it is placed in a

After such a process, the cylinder gets a permanent longitudinal magnetization that can be determined by using the proposed identification method. In order to check if the magnetic state has been correctly identified, the total induction is then extrapolated and compared to the magnetic signature measured on sensor $S_{1}$. Two identification and extrapolation algorithms have been tested MMM [4]. As already mentioned, this algorithm suffers from some inaccuracies if sensors are located too close from the sheet, as it is the case in the presented experimental set-up. The second method is the new approach proposed in this paper. Results are presented in Fig. 8.

For the MMM, although the trend is quite respected, the amplitude is clearly underestimated. The maximum of the error is $40 \%$. Additional numerical tests have shown that twice as many sensors are needed to reduce the error and achieve good anomaly prediction. The results obtained with the new method are much better. Both curves match, the mean error is less than $1 \%$. The identified $\mathbf{H}_{c}$ looks fine and is shown in Fig. 9. These results demonstrate the combination of an axial induction of $20 \mu \mathrm{T}$ and a vertical one of $40 \mu \mathrm{T}$. The cylinder is subjected to an internal pressure of $10 \mathrm{MPa}$ and then reduced to zero. It gets a permanent magnetization along both the vertical and longitudinal directions, as shown on the reconstructed $\mathbf{H}_{c}$ (Fig. 10). The field can be extrapolated at a further distance (sensor $S_{2}$ ) and compared to the measured value (Fig. 11). 
Results are also very satisfying for the identification of a more complex magnetization state. Extrapolated and measured fields are in very good accordance even if the signature level is very low (below $100 \mathrm{nT}$ at $1 \mathrm{~m}$ ). The maximal error computed is about $10 \%$ and the mean value is under $4 \%$. This last validation 265 demonstrates the efficiency of the approach.

\section{Conclusion}

A new magnetization identification algorithm based on near magnetic field measurements has been proposed. It has been applied to thin magnetic shells, described as equivalent surfaces. The approach consists in the inversion of an integral method based on the face interpolation of flux flowing within the shell. The key points of the method is the introduction of the constitutive material law in the problem and the choice of the coercive field flux as unknown. The inverse linear problem being ill-posed, a surface divergence operator has been used to change the unknowns into equivalent magnetic charges (sources of permanent

275 flux getting out of the shell). The problem becomes better-posed and can be solved by a simple balanced singular value decomposition without using any regularization technique. The efficiency of the approach has been demonstrated thanks to an experimental set-up dedicated to the study of magneto-elasticity effects, image of the ones undergone by a submarine hull. Consequently, the algorithm is well adapted in order to predict magnetic anomaly created by the ferromagnetic hull of a submarine but the method can be applied in many other areas where local permanent magnetization determination of ferromagnetic material is required.

\section{Declaration of Competing Interest}

The authors declare that they have no known competing financial interests or personal relationships that could have appeared to influence the work reported in this paper. 


\section{References}

[1] H. Igarashi, T. Honma, A. Kost, Inverse inference of magnetization distribution in cylindrical permanent magnets, IEEE Transactions on Magnetics 36 (4) (2000) 1168-1171. doi:10.1109/20.877648.

[2] L. Arbenz, O. Chadebec, C. Espanet, Y. Rtimi, G. Cauffet, Characterization of permanent magnet magnetization, IEEE Transactions on Magnetics 53 (11) (2017) 1-4. doi:10.1109/TMAG.2017.2692391.

295 [3] D. V. Lukyanenko, A. G. Yagola, N. A. Evdokimova, Applica口. tion of inversion methods in solving ill-posed problems for magnetic parameter identification of steel hull vessel, Journal of Inverse and Ill-posed Problems 18 (9) (01 Mar. 2011) 1013 - 1029. doi:https://doi.org/10.1515/jiip.2011.018. URL https://www.degruyter.com/view/journals/jiip/18/9/ article-p1013.xml

[4] O. Chadebec, J. . Coulomb, J. . Bongiraud, G. Cauffet, P. Le Thiec, Recent improvements for solving inverse magnetostatic problem applied to thin

n shells, IEEE Transactions on Magnetics 38 (2) (2002) 1005-1008. doi: $10.1109 / 20.996258$

[5] Y. Vuillermet, O. Chadebec, J. . Coulomb, L. . Rouve, G. Cauffet, J. P. Bongiraud, L. Demilier, Scalar potential formulation and inverse problem applied to thin magnetic sheets, IEEE Transactions on Magnetics 44 (6). doi:10.1109/TMAG.2007.916587.

[6] G. Jeung, C. Yang, H. Chung, S. Lee, D. Kim, Magnetic dipole modeling combined with material sensitivity analysis for solving an inverse problem

1 of thin ferromagnetic sheet, IEEE Transactions on Magnetics 45 (10). doi: 10.1109/TMAG.2009.2021853

[7] F. Bruckner, C. Abert, G. Wautischer, C. Huber, C. Vogler, M. Hinze, D. Suess, Solving large-scale inverse magnetostatic problems using the ad- 
joint method, Scientific Reports 7 (1) (2017) 40816. doi:https://doi. org/10.1038/srep40816.

[8] G. Chavin-Collin, B. Bannwarth, D. Cavallera, O. Chadebec, N. Galopin, G. Meunier, L. . Rouve, An integral face formulation for thin non-conductive magnetic regions, IEEE Transactions on Magnetics 55 (6) (2019) 1-4. doi:10.1109/TMAG.2019.2895959

[9] V. Le-Van, G. Meunier, O. Chadebec, J. Guichon, A volume integral formulation based on facet elements for nonlinear magnetostatic problems, IEEE Transactions on Magnetics 51 (7) (2015) 1-6. doi:10.1109/TMAG. 2015.2389197.

[10] P. C. Hansen, Rank-Deficient and Discrete Ill-Posed Problems, Soci1 ety for Industrial and Applied Mathematics, 1998. arXiv:https://

a epubs.siam.org/doi/pdf/10.1137/1.9780898719697, doi:10.1137/1. 9780898719697 . URL https://epubs .siam.org/doi/abs/10.1137/1.9780898719697

[11] D. Ruiz, A scaling algorithm to equilibrate both rows and columns norms in matrices, Tech. Rep. RAL-TR-2001-034, ENSEEIHT-IRIT, cM-P00040415 (September 2001).

[12] A. Viana, L. Rouve, G. Cauffet, J. Coulomb, Analytical model for external induction variations of a ferromagnetic cylinder undergoing high mechanical stresses in a low magnetic field of any orientation, IEEE Transactions on Magnetics 47 (5) (2011) 1366-1369. doi:10.1109/TMAG.2010.2078801. 

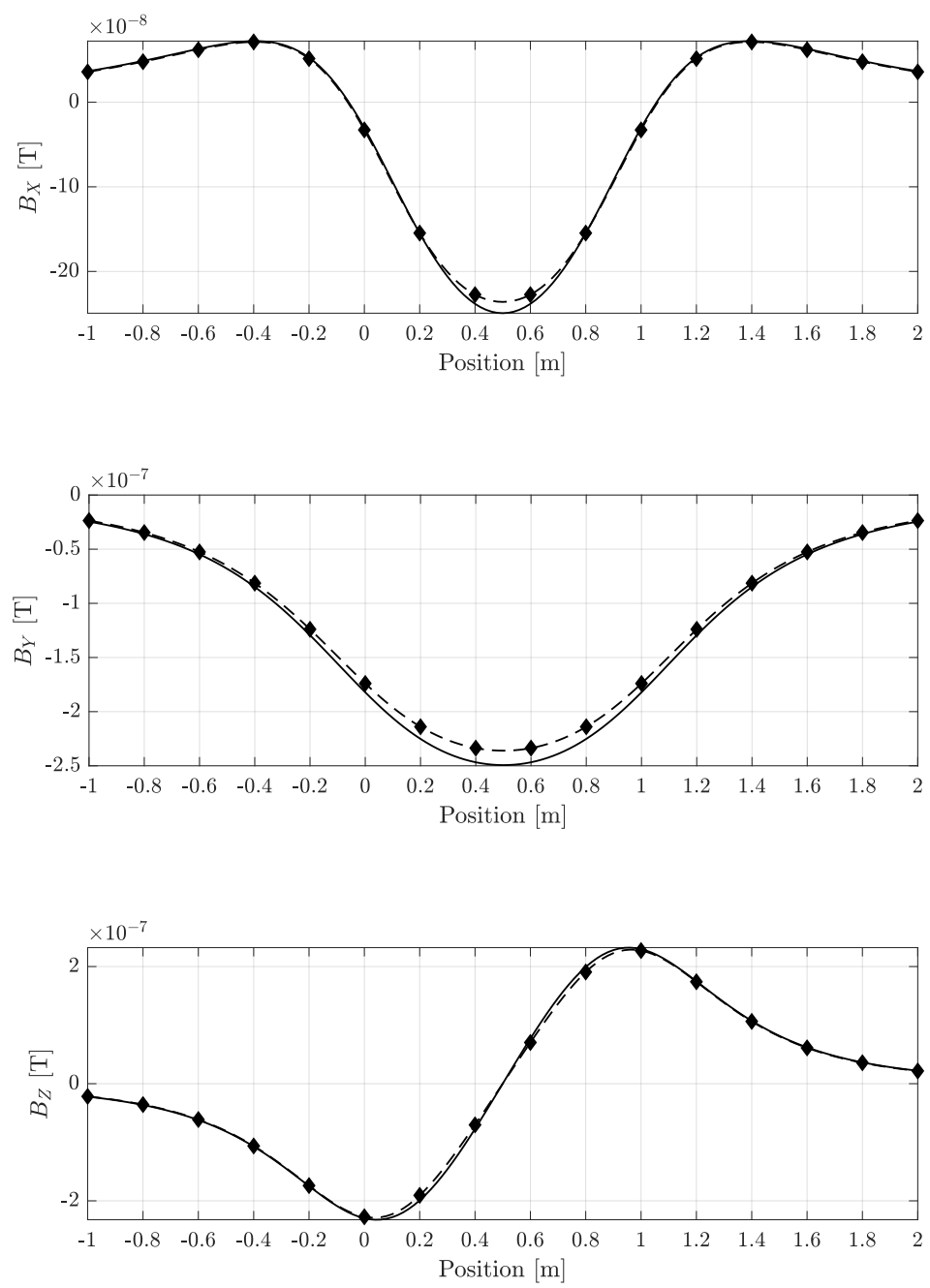

Figure 4: Comparison of magnetic induction components $(X, Y$ and $Z)$ on a line located at $0.5 \mathrm{~m}$ from the sheet - target induction (solid line, no marker) and predicted induction (dashed line, diamond markers). 


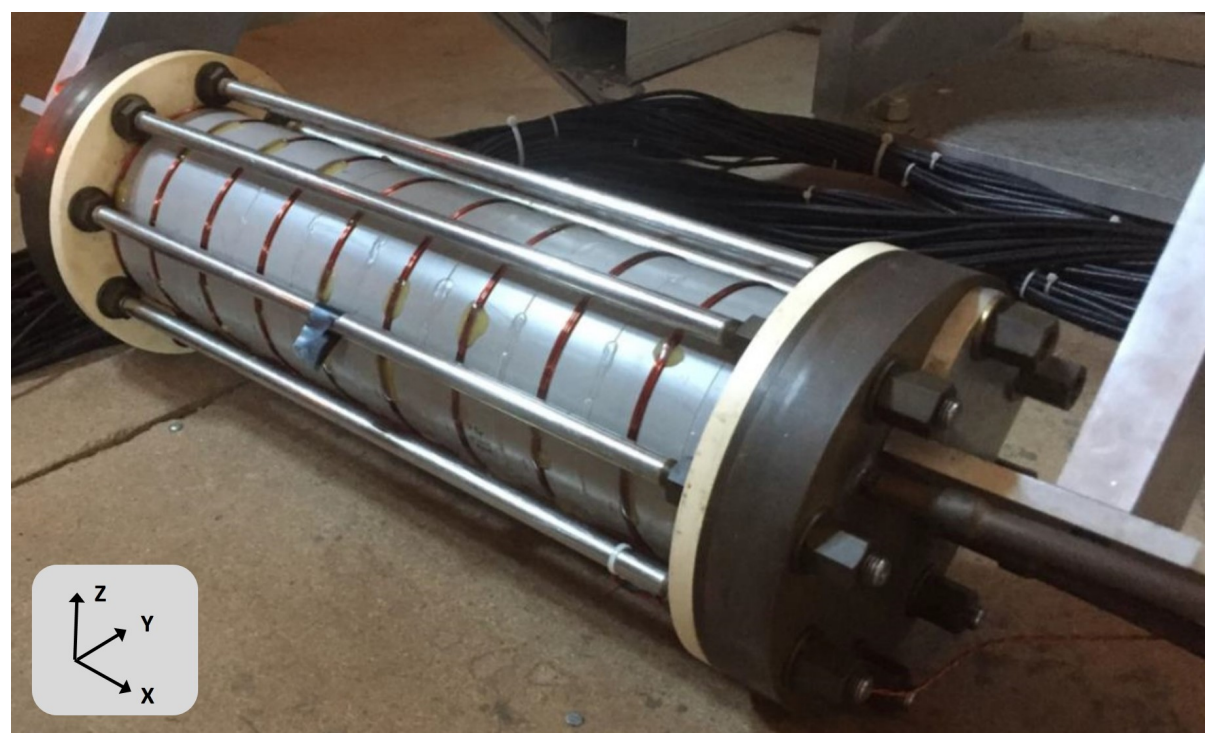

Figure 5: Hollow ferromagnetic cylinder filled by pressurized oil, with its degaussing windings. 


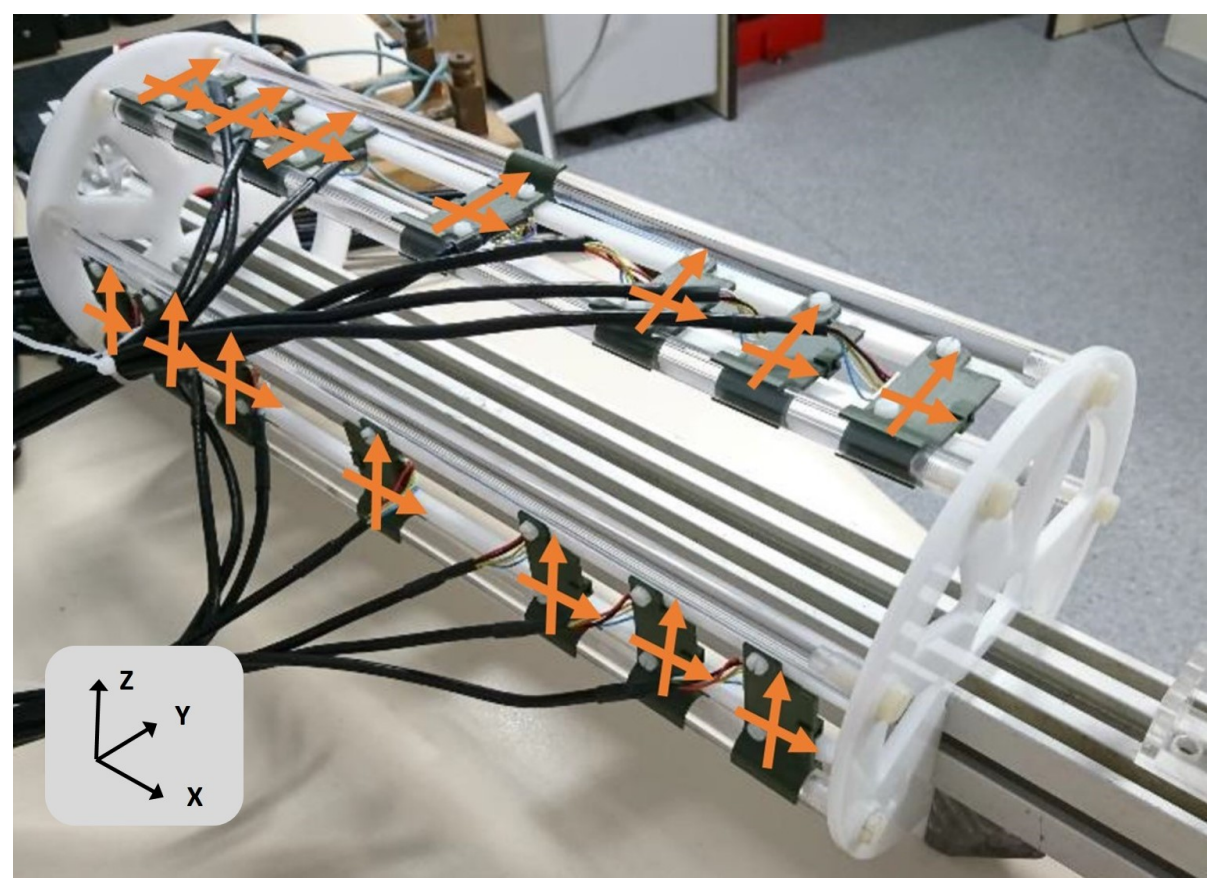

Figure 6: Mechanical support presenting the "on-board" magnetic sensors set up at $1 \mathrm{~cm}$ from the cylinder used to predict the magnetization. The cylinder does not appear on the picture. The 14 sensors are distributed along 2 lines. 


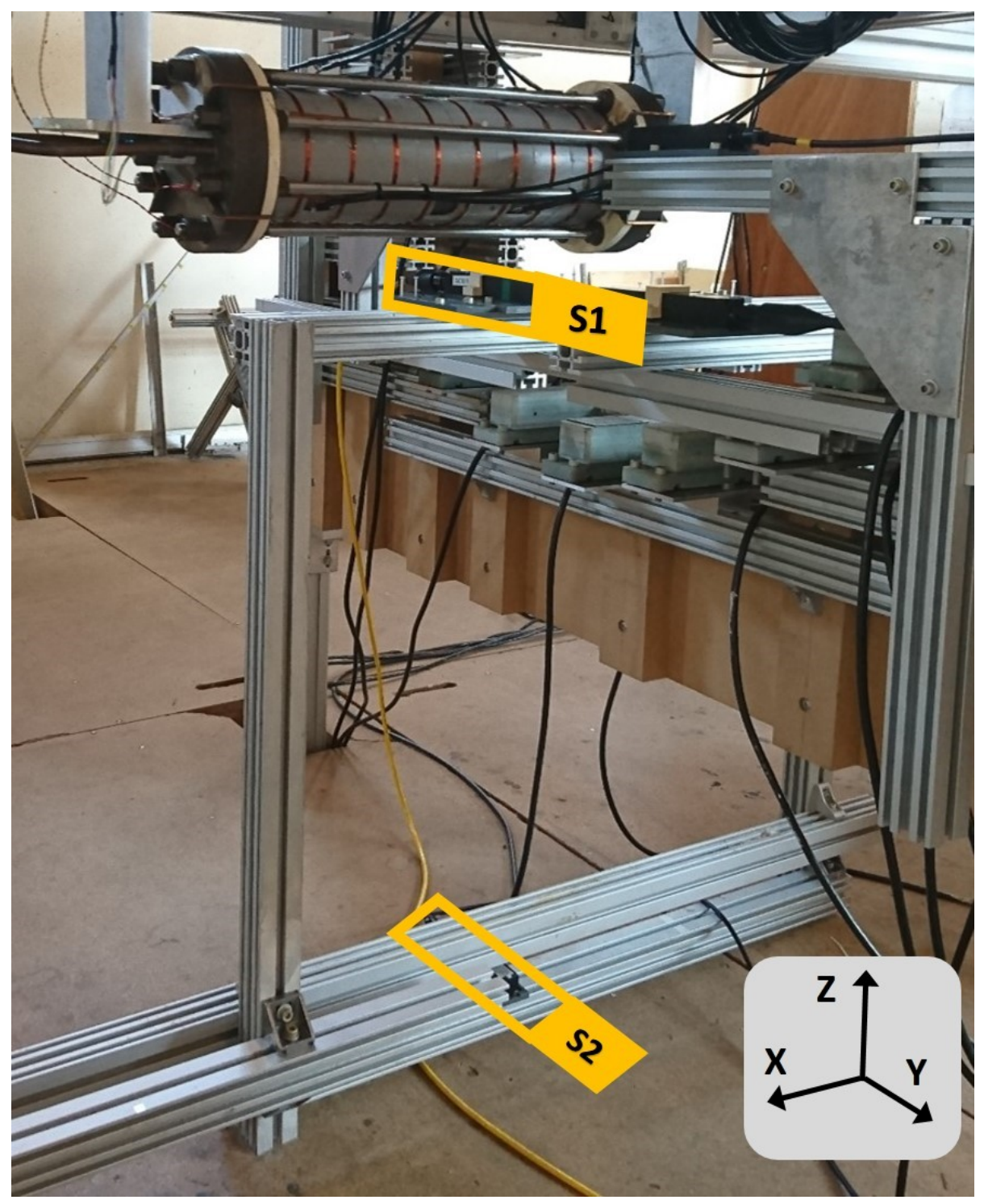

Figure 7: Cylinder mounted on the trolley with its 14 "on-board" magnetic sensors and the 2 magnetometers used for magnetic signature acquisition during the cylinder motion. 

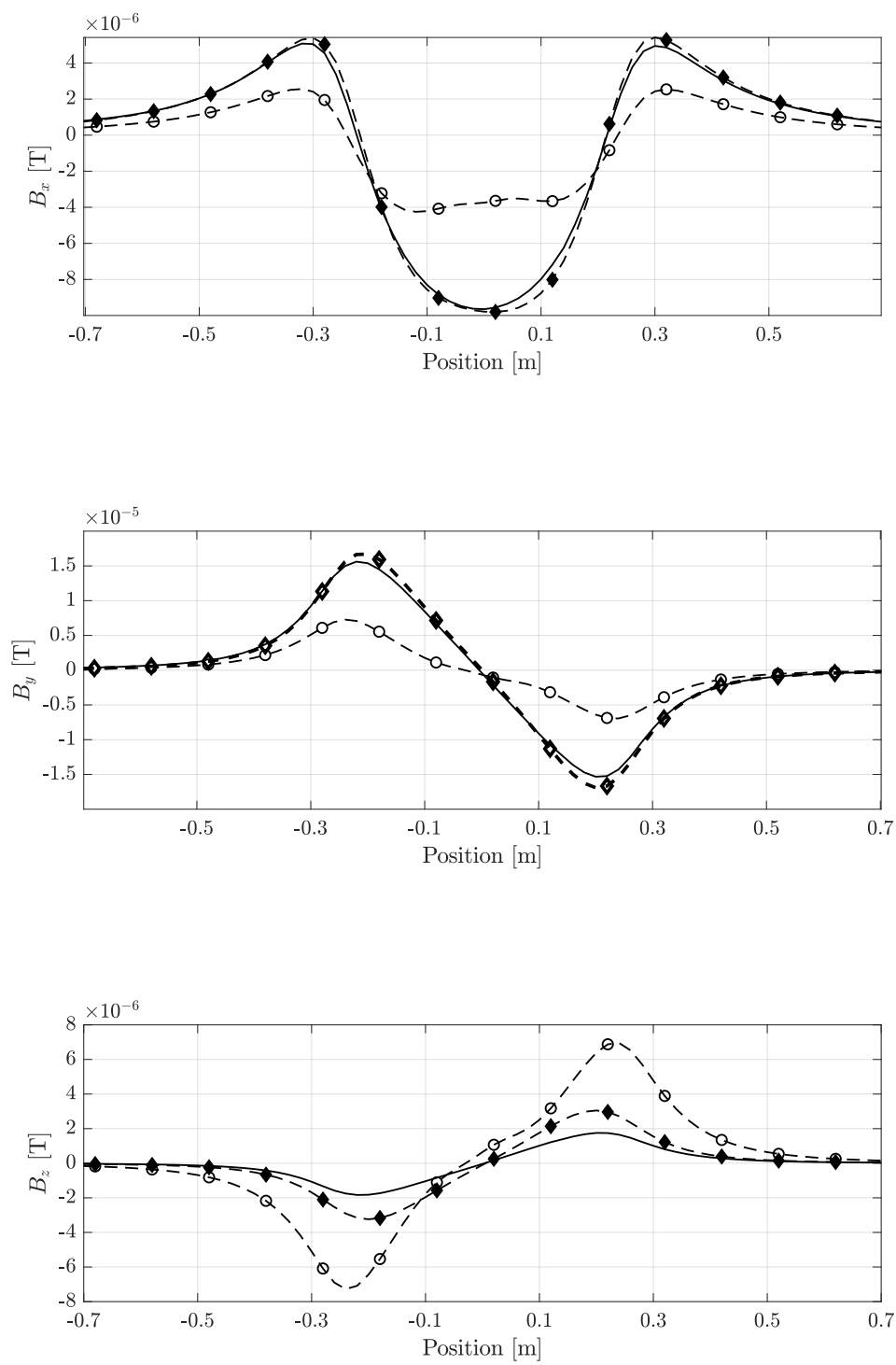

Figure 8: Longitudinal and vertical magnetic induction components on a line located at $10 \mathrm{~cm}$ below the device (sensor $S_{1}$ ) - comparison between measured induction (solid line, no marker) and predicted induction by our former 4] (dashed line, circle markers) and new algorithms (dashed line, diamond markers) for a longitudinal permanent magnetization. The reference $x=0(m)$ for the position is the middle of the cylinder. 


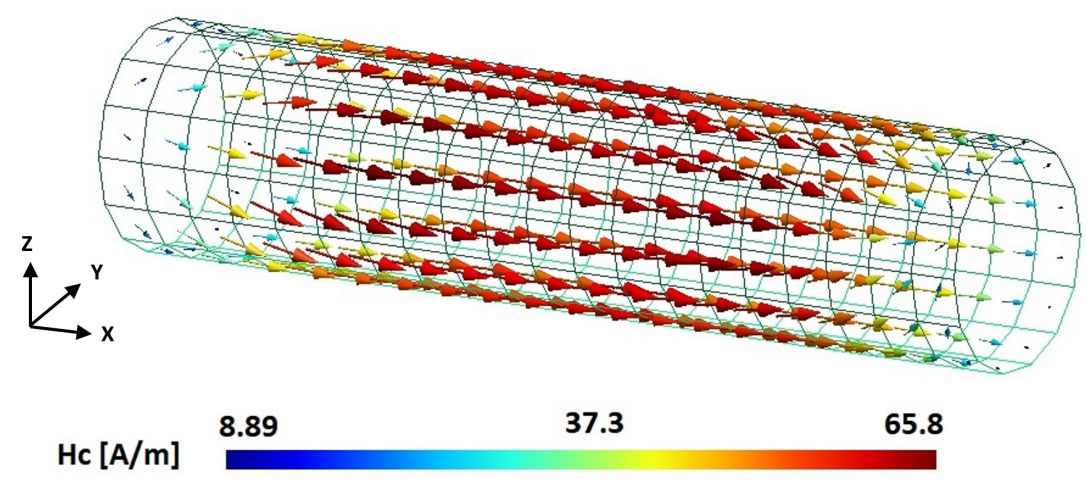

Figure 9: Reconstructed coercive field $\mathbf{H}_{c}$ inside the cylinder for the longitudinal magnetization identification.

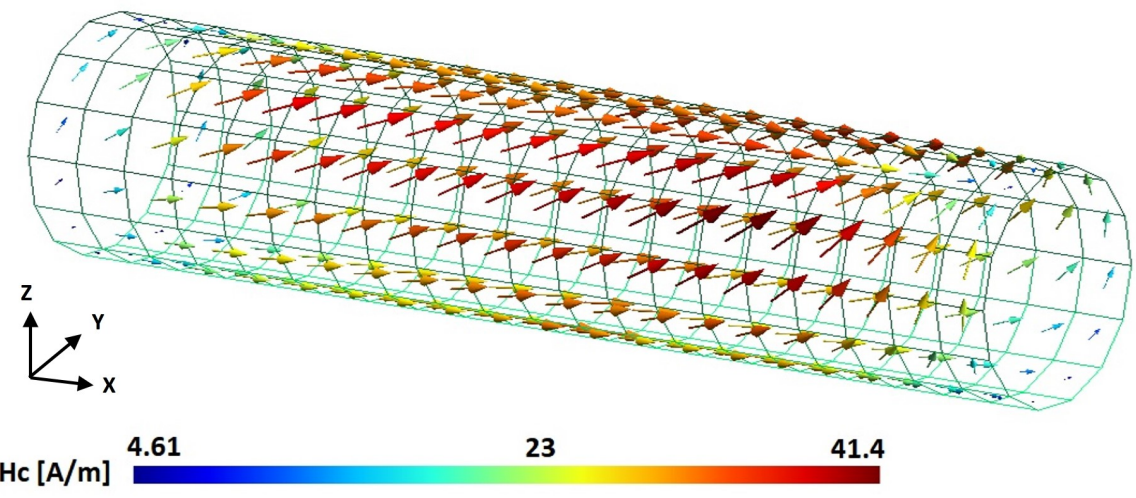

Figure 10: Reconstructed coercive field $\mathbf{H}_{c}$ inside the cylinder for the composed magnetization identification. 

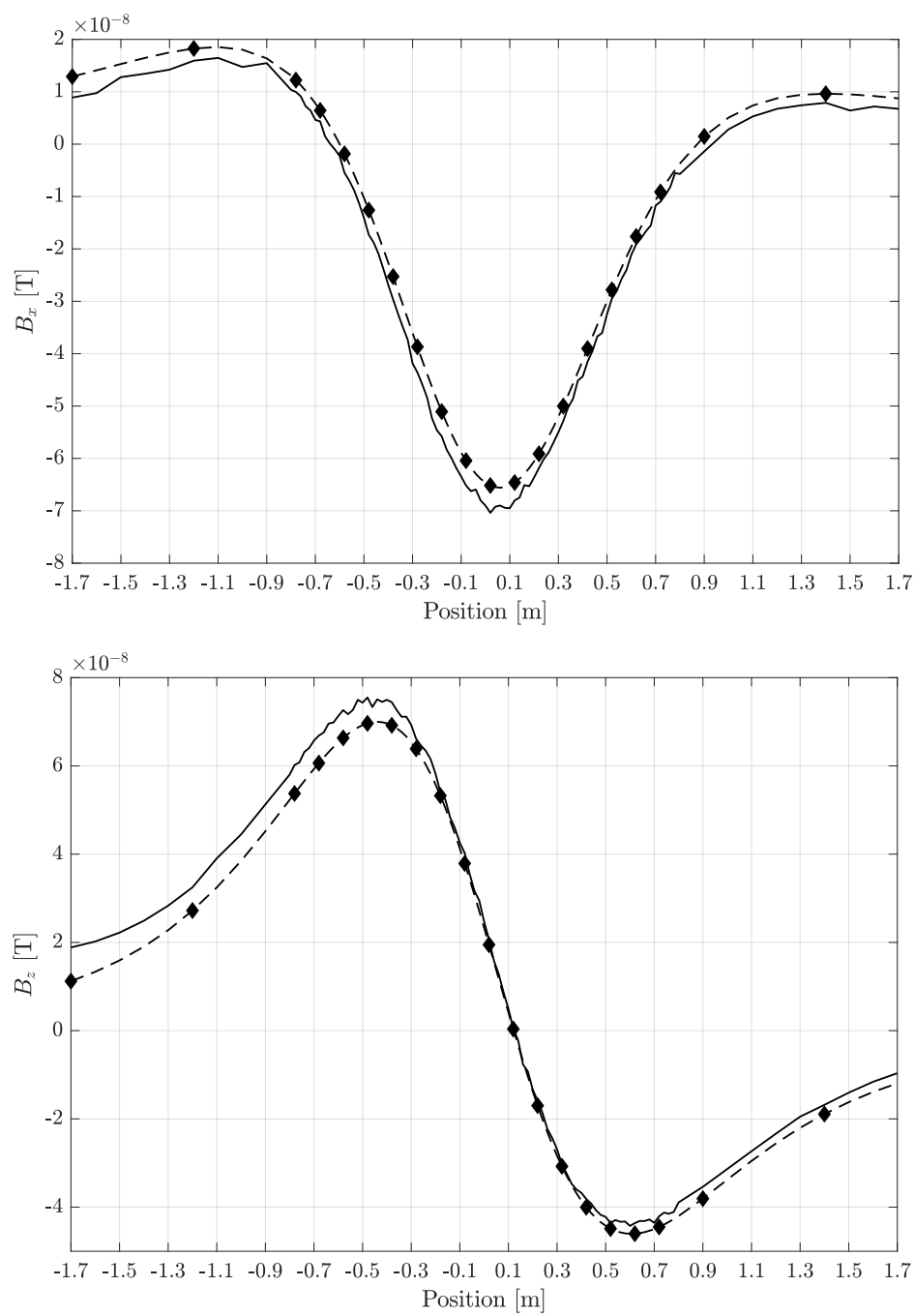

Figure 11: Longitudinal and vertical magnetic induction components on a line located at $1 \mathrm{~m}$ below the device (sensor $S_{2}$ ) - comparison between measured induction (solid line, no marker) and predicted induction (dashed line, diamond markers) for a composed permanent magnetization with both longitudinal and vertical components. 